\title{
Germanica
}

\section{Le Capitaine de Köpenick de Carl Zuckmayer : imposture et aliénation}

Karl Zuckmayers Sozialkomödie Der Hauptmann von Köpenick : Hochstapelei und Entfremdung

\section{Pierre Vaydat}

\section{(2) OpenEdition}

\section{Journals}

Édition électronique

URL : http://journals.openedition.org/germanica/1778

DOI : 10.4000/germanica. 1778

ISSN : 2107-0784

\section{Éditeur}

Université de Lille

\section{Édition imprimée}

Date de publication : 31 décembre 2004

Pagination : 11-20

ISBN : 9782913857148

ISSN : 0984-2632

\section{Référence électronique}

Pierre Vaydat, «Le Capitaine de Köpenick de Carl Zuckmayer : imposture et aliénation », Germanica [En ligne], 35 | 2004, mis en ligne le 05 octobre 2012, consulté le 06 octobre 2020. URL : http:// journals.openedition.org/germanica/1778; DOI : https://doi.org/10.4000/germanica.1778

Ce document a été généré automatiquement le 6 octobre 2020.

(c) Tous droits réservés 


\title{
Le Capitaine de Köpenick de Carl Zuckmayer : imposture et aliénation
}

\author{
Karl Zuckmayers Sozialkomödie Der Hauptmann von Köpenick : Hochstapelei \\ und Entfremdung
}

Pierre Vaydat

1 Selon Zuckmayer, le véritable sujet de sa pièce demeurée la plus célèbre est « la révolte de la créature contre la bureaucratie $»^{1}$. Thème éternel, souligne-t-il, et qui expliquerait que le succès de cette comédie ne se soit jamais démenti. Cette remarque jetée hâtivement à un journaliste est loin de laisser entrevoir les arrière-plans d'une œuvre certes mineure, mais qui a triomphé lors de sa création à Berlin et a été filmée une nouvelle fois après la seconde guerre mondiale ${ }^{2}$.

2 Zuckmayer prend quelque libertés avec la fameuse anecdote historique, dont il ne conserve en fait que certains personnages et surtout le contexte social. Dans la comédie, Wilhelm Voigt, malgré son long passé de détenu récidiviste trop lourdement condamné pour de petit méfaits, est au fond un loyal sujet de l'empereur Guillaume II. Voigt n'est pas un révolté, mais un être insignifiant, écrasé, que rend sympathique une pitoyable dignité. Or, il se trouve qu'il a l'occasion de faire à la fois l'apprentissage du comédien et du ton de commandement prussien lors de son deuxième séjour de longue durée en prison, le directeur de l'établissement étant un obsédé de la chose militaire, au point de donner aux détenus des cours de règlement et de les faire jouer sous sa direction à mimer un épisode de la bataille de Sedan auquel il a pris part et dont il cultive le souvenir impérissable. Cette propédeutique bouffonne, que Voigt accepte volontiers de subir parce qu'elle lui permet malgré tout de s'occuper l'esprit, donne au futur pseudo-capitaine les connaissances et les aptitudes indispensables à la réussite de l'imposture (dont il ne sait pas encore qu'il la commettra).

3 Car il va falloir, pour qu'elle se réalise, l'expérience de l'impossible réinsertion, et la rencontre avec l'objet magique: l'uniforme d'officier, qui n'est plus de première fraîcheur, mais qui, même chez le fripier, garde un rayonnement quasi surnaturel, la marque scintillante d'une caste de demi-dieux. L'ironie du fripier juif n'entame en rien 
la fascination qu'exerce en son veuvage l'ex-tenue du capitaine von Schlettow, bien qu'elle soit tachée en quelques endroits. "Ein deutsches Märchen » : le sous-titre de la pièce est polysémique, et le déroulement sans anicroche de l'imposture au troisième acte a la fluidité du merveilleux, celle que confère le talisman. On a noté que Voigt est lui-même un personnage de conte (Märchengestalt), car son innocence le dote d'un caractère inaltérable en dépit des épreuves qu'il traverse ${ }^{3}$.

C'est pourquoi la critique marxiste n'a guère été indulgente pour Zuckmayer : elle a perçu que le propos de l'auteur n'était pas directement didactique, qu'il ne cherchait, en fait, pas du tout à l'être ; car Zuckmayer, lui, a toujours affirmé que ses œuvres étaient de la littérature politique. Selon lui, l'intention de la pièce était claire. Il l'avait conçue dans la perspective d'un soutien qu'il aurait apporté à la République de Weimar, pour défendre la légitimité du régime démocratique contre la survivance menaçante des structures mentales wilhelmiennes ${ }^{4}$.

5 En relisant la comédie, ce qui permet une prise de distance plus aisée que lorsqu'on est pris par le charme du spectacle, on s'aperçoit que le thème de l'imposteur ne se réduit pas au seul personnage de Voigt; et l'on se prend aussi à douter que Voigt soit un imposteur véritable. Car Voigt n'est pas un délinquant ou un déviant qui s'accepte comme tel. Ce réprouvé sans noirceur ne se met que tardivement, contraint et forcé, à faire preuve de roublardise. Le déguisement n'est pour lui qu'un expédient, le contraire donc d'une attitude vitale. Il n'est qu'un moyen de se procurer le passeport refusé. Dès qu'il comprend que son entreprise a échoué parce qu'il a cru à tort que la mairie de Köpenick pouvait délivrer un tel document, il assume à nouveau sa condition de victime. Il n'est pas lié à son imposture de façon existentielle, il n'est pas un fourbe captif de sa tromperie. Tout au long de la scène où il met en arrestation le maire de Köpenick en imitant le ton cassant proverbial de l'officier prussien, il garde le contrôle ironique de son dédoublement (la scène où il sort des WC de la gare, ayant ôté son minable complet civil et revêtu l'uniforme, et réprimande un employé atterré qui, pris d'un besoin pressant, s'est rendu coupable de secouer la poignée de la porte, a servi de coup d'essai). Et la scène finale où il se démasque au commissariat le montre en train de reconquérir sa dignité. Après qu'il se soit livré, les policiers le traitent avec égards. Il lui manifestent l'estime complice due à celui qui a su mettre au jour un ridicule fondamental de l'Empire, celui de l'obéissance inconditionnelle à l'autorité militaire.

6 Car un thème symétrique est présent dès le début : celui de l'inauthenticité mentale et politique collective à laquelle s'est condamnée la bourgeoisie lorsqu'elle s'est soumise au pouvoir militaro-féodal de Bismarck et des Junkers ; le piquant de la chose étant que l'imposture n'est pas ressentie comme telle, tant l'identification au prussianisme est devenue déterminante pour l'individu. Dès lors, puisque c'est l'inconscient sociétal qui a pris le relais, il ne s'agit plus d'imposture, mais d'aliénation: les scènes les plus réussies des deux premiers actes font songer aux épisodes du Untertan, sans pouvoir toutefois rivaliser avec le comique abyssal de Heinrich Mann. La satire, chez Zuckmayer, est moins cruelle : nous entrevoyons là un autre sens du terme Märchen, à savoir la préservation de l'illusion humaniste ${ }^{5}$.

7 L'uniforme constitue le lien entre le thème de l'imposture et celui de l'aliénation. Avant de devenir le support de l'imposture, il est le symbole évident d'une discipline qui exige de ses représentants qu'ils assument la dépersonnalisation.

8 Le capitaine von Schlettow se confond volontairement avec son rôle idéaltypique, il a la chance d'être membre d'une caste ayant éliminé le mal d'être, puisque, dans son mode 
de vie, le devoir et l'inclination coïncident. Les observations qu'il fait au tailleur sur le placement des boutons au bas du dos, et qu'il conclut de façon carrément philosophique, montrent qu'il s'est aliéné totalement à l'ordre prussien, armature d'une personnalité synthétique, qui ne réalise ses aspirations qu'en se conformant aux détails les plus infimes du règlement. À l'inverse, le moindre manquement à la présentation requise lui cause une gêne morale :

Lachense nicht Wabschke. Sie denken, das is ne Kleinigkeit. Is auch ne Kleinigkeit. Aber an den Kleinigkeiten erkennt man den Soldaten. Darauf ist alles aufgebaut, da steckt $\mathrm{n}$ tieferer Sinn drin, verstehnse ${ }^{6}$

9 Le «sens profond" est l'indice de l'intériorisation accomplie, récompensée par le sentiment de plénitude et de puissance vitale dont l'uniforme porté de façon impeccable investit son détenteur.

Or, c'est précisément cette intériorisation complète qui va provoquer la chute de von Schlettow, la fin prématurée de sa carrière. Pour jouer au billard avec son ami, le docteur Jellinek, dans un café plutôt louche de la Friedrichstrasse fréquenté par des grisettes, Schlettow a recours à une imposture que d'aucuns jugeraient sans gravité, tant elle est quotidienne : il s'est mis en civil pour ne pas être remarqué. Il est conscient néanmoins du danger qu'il court en ayant pris la liberté de venir dans ce lieu inconvenant, qu'un officier prussien devrait s'interdire de fréquenter :

[...] für Militär verboten - eigentlich. Sehnse - das müssense sich mal vorstellen unsereins - Offiziersfamilie seitm Siebenjährigen Krieg [...] da is man nu in puncto Firmenschild verdammt aufm Kasten. Offiziersberuf is dauernd öffentliche Verantwortung, hat mein oller Herr immer gepredigt - und damit hat ers bis zum Kommandierenden General gebracht. Aufn bunten Rock kein Stäubchen - das is mir Lebensaufgabe ${ }^{7}$.

11 En cédant à son goût du billard et en mentant par son déguisement concernant son appartenance, il a failli à cette obligation d'intégrité. La punition du destin ne tarde pas. Il intervient imprudemment, oubliant la personnalité de consommateur ordinaire qu'il a provisoirement assumée, dans une bagarre opposant pour une prostituée un grenadier ivre et un repris de justice. Lui et le grenadier sont emmenés au poste par un schupo appelé en renfort. Comble d'humiliation, Schlettow doit en outre subir les propos injurieux des badauds, qui lui en veulent d'avoir agressé un militaire de la Garde. Il ne lui reste plus qu'à démissionner de l'armée et à formuler pour son brosseur apitoyé la morale de cette histoire :

Quasselnse nich, Deltzeit. So viel Pech darf $\mathrm{n}$ Soldat nich haben, das is es. Unglück is auch $n$ Versagen. Schluss jetzt ${ }^{8}$.

Dans l'économie de la pièce, von Schlettow devient le faire-valoir de Voigt, qui achète l'uniforme rendu au tailleur, puis devenu moins éclatant à force de tribulations, et qui a fini par échouer dans un magasin de défroques9. Von Schlettow est devenu la victime du système psychorigide, tandis que Voigt l'incarne à s'y méprendre.

13 Au début du deuxième acte ${ }^{10}$, nous voyons Voigt prouver qu'il a assimilé de façon parfaite l'instruction militaire dispensée aux détenus de la centrale de Sonnenburg par le Zuchthausdirektor, dont cette éducation censément régénératrice est la marotte. Pour complaire à ce dernier, le détenu Voigt joue le rôle d'un officier lors d'une pantomime grotesquement dérisoire et bâclée, censée reproduire un épisode de la bataille de Sedan. Félicité par le directeur pour avoir commandé avec compétence, Voigt arbore sa prédisposition à l'imposture en prenant obséquieusement la pose patriotique: 
Das hat $\mathrm{n}$ Preusse im Blut, Herr Direktor. communal Obermüller ${ }^{13}$. Il s'agit de ce même Obermüller qui, devenu bourgmestre au troisième acte, sera la dupe de Voigt mué en imposteur. Obermüller vient de réaliser l'une de ses principales ambitions : devenir sous-lieutenant de réserve. Bien que libéral de gauche (il est membre de la Fortschrittliche Volkspartei), Obermüller accepte toutes les conventions sociales et idéologiques du système. Ayant besoin d'un uniforme correspondant à son nouveau grade, il se laisse convaincre par Wormser d'endosser celui que von Schlettow a rendu, et auquel le coupeur va apporter quelques retouches. Pendant que l'on s'affaire autour de lui, Obermüller tient des propos tout aussi conformistes que ceux émis par Wormser précédemment, mais se situant à un niveau plus élevé, celui de l'idéalisme bien-pensant exigé du fidèle sujet, tirade où transparaît, par le même procédé d'accumulation des clichés, l'imposture objective du régime imposé par Bismarck :

Das Grosse ist bei uns die Idee des Volksheeres, in dem jeder Mann den Platz einnimmt, der ihm in der sozialen Struktur der Volksgemeinschaft zukommt! 'Freie Bahn dem Tüchtigen!' Das ist die deutsche Devise! Die Idee der individuellen Freiheit verschmilzt bei uns mit der konstitutionellen Idee $\mathrm{zu}$ einem entwicklungsfähigen Ganzen. Das System ist monarchisch - aber wir leben angewandte Demokratie! Das ist meine Überzeugung!

L'imposture de Voigt ridiculise cette omniprésence écrasante du système prussien, mais elle n'est pas un acte révolutionnaire. Elle est tout au plus une mystification moralement justifiée. Au dénouement, aucune modification n'est intervenue dans le rapport de pouvoir. Voigt n'a pas cherché une revanche, mais une issue. Au sortir d'un 
épisode burlesque, il demeure un personnage de comédie, émouvant parfois, mais qui n'est susceptible d'aucun grandissement. Ce malchanceux n'a pas la malhonnêteté épique, gouailleuse, du personnage picaresque. C'est pourquoi le pouvoir le traite, lorsqu'il se rend à la police après avoir manqué son coup, avec une indulgence amusée. $\mathrm{Au}$ commissariat, une fois qu'on a constaté très vite qu'il est inoffensif, on lui offre le porto. (Dans l'histoire réelle, le Kaiser l'a gracié au bout de vingt mois d'emprisonnement, point mécontent au fond d'un incident qui avait démontré l'esprit discipliné de ses sujets et la solidité apparemment inébranlable de la monarchie militaire.) La portée politique de l'incident a donc été nulle. L'affaire de Saverne, sept années plus tard, a constitué une fissure morale autrement sérieuse, comme en ont témoigné les bruyantes manifestations d'irrespect au Reichstag envers le chancelier Bethmann Hollweg et la motion de défiance votée contre lui à une écrasante majorité. Mais, dans ce cas également, le semi-absolutisme prussien a survécu au ridicule. Les conservateurs ne tireront de l'épisode aucun enseignement, bien au contraire. C'est ainsi que le député conservateur Elard von Oldenburg-Januschau, accentuant par défi sa morgue de Junker autocratique, lancera le 29 janvier 1910 en plein Reichstag, en se réclamant de la «tradition prussienne »:

Der König von Preussen und der Deutsche Kaiser muss jeden Moment imstande sein, zu einem Leutnant zu sagen : 'Nehmen Sie zehn Mann und schliessen Sie den Reichstag ! ${ }^{14}$

Il a fallu que le système prussien tombe dans le discrédit moral complet après l'effondrement militaire de 1945 pour que le regard critique surévalue démesurément l'importance de l'incident, comme en témoignent ces lignes extraites d'un ouvrage par ailleurs d'un bon niveau :

Als an einem Herbsttag des Jahres 1906 der siebenunfünfzigjährige Schuster und langjährige Zuchthäusler in der Uniform eines preussischen Hauptmanns mit zehn aufgegriffenen Soldaten das Rathaus von Köpenick besetzte und sich mit der Staatskasse davonmachte, da lachte die Welt über einen Militär- und Polizeistaat, dessen gebrechliches System ein Schelm demaskiert und dem Gespött ausgeliefert hatte. Der Streich des Hauptmanns von Köpenick war die deutsche Halsbandaffäre des 20. Jahrhunderts: auf seine Art ein welthistorisches Ereignis, das die veräusserlichte Formalität eines auf Obrigkeitsdenken und Untertanengeist gegründeten Staatswesens entblösste ${ }^{15}$.

Envisagé sous l'angle de l'objectivité historique, le fait divers amusant de Köpenick n'a préludé à aucun changement. Et dans la fiction théâtrale de Zuckmayer, le rire énorme qui s'empare de Voigt, puis des policiers, paraît plaqué, sans vertu libératrice. Les spectateurs de 1931 pressentent l'avènement proche d'un nouveau régime militariste bien plus oppressif que celui de Guillaume II, et dans lequel l'imposture plaisante n'aura plus la place que pouvait encore lui accorder un environnement social et politique au conformisme certes plein de raideur, mais demeurant relativement humain malgré tout. Le critique Paul Rilla le soulignait en 1950 :

Welches Gelächter weckt die Komödie Zuckmayers? Schon vor sechzehn Jahren konnte man erkennen, dass dies kein satirisches Zeitbild, sondern eine Illuminierung jenes preussischen Idylls war, dessen Frostigkeit durch Zuckmayers süddeutsches Geblüt erwärmt wird, bis die Kahlheit gemütvoll zu blühen beginnt [...] Zuckmayer zeichnet die subalterne Unmenschlichkeit der bürokratischen Maschinerie, in deren Räderwerk der Schuster Voigt gerät. Aber die subalternen Repräsentanten zeichnet er als die Arrivierten einer Beschränktheit, die eher liebenswert als verabscheuungswürdig wirkt ${ }^{16}$. 
Aux yeux de P. Rilla, critique marxiste, la pièce de Zuckmayer, pour être talentueuse, ne présente pas au fond un véritable intérêt, parce qu'il estime qu'elle invite à l'évasion au lieu d'inciter à l'action :

Im Hauptmann von Köpenick bot (Zuckmayer) die zeitliche Distanz zu dem kuriosen Vorfall von 1906 die Möglichkeit, sich aus der Satire in ein Kuriositätenkabinett zurückzuziehen ${ }^{17}$.

La comparaison avec le Révizor de Gogol, pièce fameuse sur le thème de l'imposteur, peut cependant permettre de corriger quelque peu le reproche de superficialité fait à Zuckmayer. En effet, bien qu'elle soit antérieure d'un siècle à la comédie berlinoise sur le faux capitaine, celle de Gogol se déroule dans un contexte social et politique similaire par bien des aspects, encore que l'autocratie russe de ce temps-là ait été un régime beaucoup plus cruellement (et comiquement) arriéré que ne le sera le semi-absolutisme du Reich prusso-allemand. La ressemblance thématique est indéniable, et va plus loin qu'une simple parenté de sujet. Car chez Gogol également, des piliers subalternes de la société se laissent prendre à la fausse qualité d'un personnage apparemment nimbé d'une autorité sans limites. Il s'agit en l'occurrence de l'inspecteur général de l'administration dont les autorités municipales attendent l'arrivée incognito, et avec lequel le médiocre dandy Khlestakov, obligé par la dèche d'interrompre son voyage, est confondu. Dans les deux pièces satiriques, l'imposture réussit parce que les rapports de pouvoir et la mentalité collective lui offrent un terrain propice. A la différence de l'escroquerie, l'imposture est dépendante d'une certaine immobilité politique et sociale. La Russie de Nicolas $\mathrm{I}^{\text {er }}$ connaît en 1836 une paix profonde et une stabilité proche de la stagnation. En 1906, malgré la première crise marocaine et le rivalité navale avec l'Angleterre, l'éventualité d'une guerre suivie de bouleversements sociaux paraît lointaine ${ }^{18}$. L'incident a égayé le Kaiser :

INSPEKTOR : Der Kaiser is gar nich so scharf auf die Sache. Im Gegenteil! Habense den Geheimbericht nich gelesen? Gelacht hat er, wie man's ihm vorgetragn hat, und stolz war er noch drauf! Mein lieber Jago (sic), hat er zum Präsidenten gesagt, da kann man sehen, was Disziplin heisst ! Kein Volk der Erde macht uns das nach ${ }^{19}$. Attitude identique chez Nicolas $\mathrm{I}^{\mathrm{er}}$ cent ans plus tôt. On lit chez Herzen :

Der Kaiser Nikolaus schüttelt sich vor Lachen bei der Aufführung des « Revisors »!!! [...] Allerhöchste Heiterkeit [...] süffisantes Lächeln der Beamten $[\ldots]^{20}$

Les significations des deux comédies sont donc parallèles, sans être identiques ${ }^{21}$. L'imposture d'un seul démasque chez Gogol l'hypocrisie de tous, chez Zuckmayer l'aliénation de chacun. Dans le Révizor, le tableau final nous montre l'ensemble des personnages pétrifiés lorsqu'ils comprennent qu'ils ont été joués et que le directeur de la poste locale, friand de décacheter, leur annonce l'arrivée imminente et vraisemblablement catastrophique du véritable inspecteur. Le rire monstrueux de Voigt et des policiers au dénouement du Hauptmann indique lui aussi que tout reste en suspens dans une société bloquée («Unmöglich!", le mot de la fin, prend vu sous cet angle tout son sens). 


\section{NOTES}

1. Voir son interview dans l'hebdomadaire suisse Die Weltwoche, donné quelques mois avant sa mort : « Als wär's ein Stück von ihm », 22.12.1976.

2. Par Helmut Käutner, en 1956, avec Heinz Rühmann dans le rôle principal.

3. Cf. Paul Meinherz, Carl Zuckmayer. Sein Weg zu einem modernen Schauspiel, thèse Univ. de Zurich, 1960.

4. Cf. ce que dit C.Z. lui-même dans son livre de souvenirs, Als wär's ein Stück von mir, Fischer Taschenbuch Verlag, 1977 (1 ère éd. 1969), p. 375 : « Die Wirkung des Hauptmann von Köpenick war tiefer und nachhaltiger als die des Fröhlichen Weinberg. Das Stück wurde, von Freund und Feind als das Politikum begriffen, als das es gemeint war. [...] Gerade dass hier auch die 'Gegenseite', das Militär vor allem, nicht blindlings verdammt und verteufelt, sondern mit dem Versuch zur dramatischen Gerechtigkeit dargestellt wurde, machte das Stück und sein Anliegen glaubwürdiger und liess nicht das Misstrauen und den üblen Nachgeschmack aufkommen, den betonte, einseitige Tendenz oder 'Propaganda' immer verursacht. »

5. La part de la tendresse humaine dans le roman de Heinrich Mann est très réduite : elle se limite à la description de la brève amourette entre Agnes Göppel et Diederich, parodie des scènes d'amour chez Fontane; encore ce dernier n'est-il pas sincère et rompra-t-il d'une manière ignoble. En revanche, chez Zuckmayer, Voigt est accueilli avec humanité, puis avec affection, par sa soeur et son beau-frère (Marie et Friedrich Hoprecht), bien qu'il ne leur ait pas donné signe de vie depuis des années. Et Voigt s'occupe avec une émotion vraie de la jeune fille mourante qu'ils abritent, épisode non exempt de sentimentalité facile.

6. Acte I, scène 1 .

7. Acte I, scène 3.

8. Acte I, scène 5 .

9. Acte III, scène 15 .

10. Acte II, scène 8.

11. Acte I, scène 1.

12. Ibid.

13. Acte I, scène 7.

14. Cité par Heinrich August Winkler, Der lange Weg nach Westen, I, Deutsche Geschichte vom Ende des alten Reiches bis zum Untergang der Weimarer Republik, Beck, München, 2000, p. 310.

15. Klaus Hermsdorf, Thomas Manns Schelme, Rütten und Loening, Berlin, 1968, p. 14-15.

16. "Zuckmayer und die Uniform », in Literatur, Kritik und Polemik, Verlag B. Henschel und Sohn, Berlin, 1950, p. 9.

17. Ibid., p. 11.

18. Cf. Acte III, scène 21 .

19. Acte III, scène 21 .

20. Alexander Herzen, Russlands soziale Zustände, $1^{\text {ère }}$ édition : Hoffmann und Campe, Hambourg, 1854 ; rééd. Reclam, Leipzig, 1921, p. 145.

21. C'est peut-être la raison pour laquelle Thomas Mann a songé au rapprochement, mais d'une manière qui peut paraître hyperbolique et désagréablement flagorneuse : « Seit Gogols Révisor die beste Komödie der Weltliteratur » hiess es in einem spontanen Brief von Thomas Mann, den er mir nach dem Besuch der Aufführung geschrieben hatte. » (Als wär's ein Stück von mir, ibid.). 


\section{RÉSUMÉS}

On considère d'habitude Le Capitaine de Köpenick comme une simple satire, tantôt sentimentale, tantôt amusante du militarisme prussien et de son emprise sur la société wilhelmienne. Le soustitre de la pièce indique que c'est avec l'assurance merveilleuse du héros des contes que Willhelm Voigt, l'imposteur fondamentalement plein de bonté, triomphe du principe d'autorité prussien, qui a fait de lui des années durant une victime de l'inhumanité sociale. Notre analyse se propose de montrer que Zuckmayer, par son traitement dramatique d'une imposture plutôt cocasse malgré son arrière-plan tragique, est parvenu à dénoncer le contexte d'aliénation caractérisant l'époque de Guillaume II. La comparaison faite par Thomas Mann avec le Révizor de Gogol, exagérément louangeuse à première vue, peut se justifier sous cet angle.

Gemeinhin wird Karl Zuckmayers Hauptmann von Köpenick bloss als eine teils empfindsame, teils vergnügliche Satire auf den preussischen Militarismus und seine Durchdringung der wilhelminischen Gesellschaft betrachtet. Das Märchenhafte, worauf der Untertitel der Komödie hindeutet, besteht in der selbstsicheren Weise, wie der im Grunde herzensgute Schwindler Wilhelm Voigt das preussische Autoritätsprinzip überwindet, das ihn lange Jahre hindurch zum Opfer der Inhumanität gemacht hatte. Diese Analyse will aufzeigen, dass es Zuckmayer gelungen ist, durch die Dramatisierung eines an sich trotz seines tragischen sozialen Hintergrunds eher possenhaften Vorgangs den Wilhelminismus als Entfremdungszusammenhang hinzustellen. Unter diesem Blickwinkel lässt sich Thomas Manns Lobpreisung - die auf den ersten Blick reichlich übertriebene Gleichsetzung mit Gogols Revisor - vielleicht doch einigermassen rechtfertigen.

\section{AUTEUR \\ PIERRE VAYDAT}

Université Charles-de-Gaulle - Lille 3 